\title{
Diagnostic, genetic and therapeutic challenges in mixed phenotype acute leukemia: A case report
}

Noran Qawasmeh', Mohammed Abu-Rayyan², Mamdouh Skafi², Moien Atrash², Ibrahim Yaghmour ${ }^{3}$, Ahlam Mujahed ${ }^{3}$, Hamdi AlJanazreh $^{2 *}$ and Fortunato Morabito ${ }^{2,4}$

${ }^{1}$ College of Medicine, Al-Quads University, Abu-Dies, Palestine

${ }^{2}$ Hematology Department and Bone Marrow Transplant Unit, Cancer Care Center, Augusta Victoria Hospital, East Jerusalem, Israel

${ }^{3}$ Laboratory Department, Augusta Victoria Hospital, East Jerusalem , Israel

${ }^{4}$ Biotechnology Research Unit, Aprigliano, Cosenza, Italy

\section{Introduction}

Mixed Phenotype Acute Leukemia (MPAL) is recognized by the World Health Organization (WHO) classification as a rare subtype of acute leukemia (AL) of multilineage origin [1], comprising $1-3 \%$ of leukemia worldwide $[2,3]$. The estimated annual incidence is of 0.35 cases per 1,000,000 person-years [3]. MPAL diagnosis is based on the combined expression of lymphoid, either B- or T-, and myeloid markers, potentially showing a $\mathrm{B} / \mathrm{T}$ lymphoid, $\mathrm{B} /$ myeloid, $\mathrm{T} /$ myeloid, or even and more rarely, a trilineage $\mathrm{B} / \mathrm{T} /$ myeloid commitment [1]. In addition, WHO recognizes two distinct categories: MPAL with the $\mathrm{t}(9 ; 22)(\mathrm{q} 34 ; \mathrm{q} 11) / \mathrm{BCR}-\mathrm{ABL} 1$ and MPAL with translocations involving the 11q23 breakpoint (MLL gene) rearrangement (MLLr). Roughly 80 different partner genes have been described [4]. The ALL1-fused gene from chromosome 4 (AF4) is fused in-frame to MLL as a result of a $\mathrm{t}(4,11)(\mathrm{q} 21, \mathrm{q} 23)$ translocation [2] in about $50 \%$ of infant ALL and in $>75 \%$ of adult MLLr AL [4]. Notably, the gene expression signature of MLLr is distinct from all other AL subtypes, with HOX cluster genes and the HOX cofactor MEIS1 being the most frequently overexpressed genes, whose deregulated expression could explain the stem cellassociated characteristics of MLLr AL, conferring to the leukemic cells oncogenic properties and survival advantages, ultimately leading to a dismal clinical outcome.

The rarity of MPAL, with or without MLLr, makes it difficult to establish the best therapeutic approach for these patients. However, ALL-oriented treatment seems more effective with a higher response rate and better outcome than an AML schedule [3,5]. Herein, we report a rare $\mathrm{B} / \mathrm{T} /$ myeloid MPAL case with MLL-AF4 gene rearrangement, successfully treated with an AML-oriented protocol, after failing leukemic blast clearing as a result of pre-phase steroid treatment.

\section{Case presentation}

A 34-year-old Palestinian social worker was admitted at the Augusta Victoria Hospital on September 2018 because of pancytopenia, multiple bluish-to-purple skin lesions, gingival bleeding, fever and night sweats. Blood cell count showed WBC $47 \times 10^{3}$, HGB $9 \mathrm{~g} / \mathrm{dL}$, and platelets $19 \times 10^{3}$. The anal swab was positive for extended-spectrum beta-lactamase (ESBL) Escherichia coli and carbapenem-resistant enterobacteriaceae (CRE). Bone marrow (BM) aspiration and biopsy showed a diffuse infiltration of blasts displaying ALL morphology. Flow cytometry analysis (Figure 1) indicated blast population positive for CD19 (52\%), CD33 (32\%), CD15 (60\%), CD11b (30\%), CD11c (32\%),
CD64 (49\%), cytoplasmic MPO (42\%), and cytoplasmic 79a (27\%), while negative for CD20, CD25, CD10, CD7, CD3, CD34, CD117, CD56, CD13, and CD14. Monoclonal gene rearrangement of both Tand B-cell receptors were also demonstrated. RT-PCR revealed MLLAF4 gene rearrangement, while BCR-ABL, CBFB (INV16), AML1ETO, NPM1, PML-PARA, and FLT3 were negative. Cerebrospinal fluid analysis was negative. Overall, these results fulfilled the criteria of the 2008/2016 WHO classification system of MLL-AF4-rearranged MPAL with trilineage (B/T/myeloid) commitment.

Baseline whole body computed tomography (CT) showed bilateral atypical cavitary lung lesions suggestive of invasive fungal infection (Figure 2A). The patient was empirically started on voriconazole. Ten days later, the patient showed clinical and radiological improvement (Figure 2B). Since literature data suggest ALL-oriented treatment for MPAL patients $[3,4]$, a pre-phase therapy with steroid was administered for a week. However, minimal response on peripheral blast count was appreciated. Considering the failed response to steroid therapy, an additional negative prognostic indicator, the patient was switched to an AML-based protocol, consisting of idarubicin $12 \mathrm{mg} / \mathrm{m}^{2}$ for 3 days associated with cytarabine $100 \mathrm{mg} / \mathrm{m}^{2}$ continuous infusion on days 1-7 and etoposide $100 \mathrm{mg} / \mathrm{m}^{2}$ on days 1-5. Bone morrow aspiration and biopsy performed at day 35 showed complete remission (CR) (blast cell $<5 \%)$.

The patient was readmitted two weeks later for consolidation chemotherapy with high dose cytarabine (HIDAC) $3000 \mathrm{mg} / \mathrm{m}^{2}$ days 1,3 , and 5 . The patient developed febrile neutropenia with a positive blood culture for ESBL E.coli, successfully treated with meropenem and vancomycin. A second cycle of HIDAC consolidation chemotherapy was administered after a further BM evaluation confirming CR (2.5\% blast cells). The patient experienced febrile neutropenia with CRE positivity on blood culture. Ceftazidime, amikacin, and colistin successfully resolved the infection. A third cycle of HIDAC consolidation chemotherapy, with a further episode of febrile neutropenia, successfully treated using

${ }^{\star}$ Correspondence to: Hamdi Al-Janazreh, Hematology Department and Bone Marrow Transplant Unit, Cancer Care Center, Augusta Victoria Hospital, East Jerusalem, Israel, Tel: +970599129800; E-mail: Dr_hamdi@hotmail.com

Key words: Mixed phenotype acute leukemia, MLL(AF4)-rearrangement, chemotherapy, carbapenem-resistant enterobacteriaceae (CRE) infection

Received: May 13, 2019; Accepted: May 30, 2019; Published: June 03, 2019 


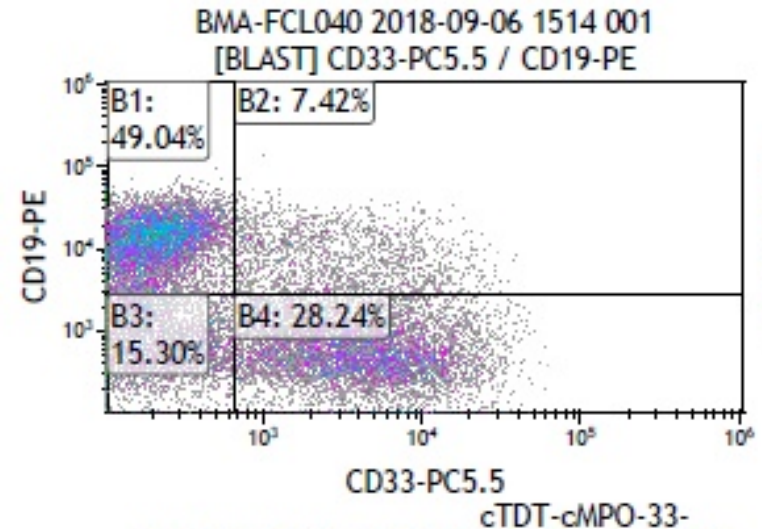

c22-45KO BMA-FCL040 2018-09-06 1538 001

[BLAST] cTDT-FITC / cMPO-PE

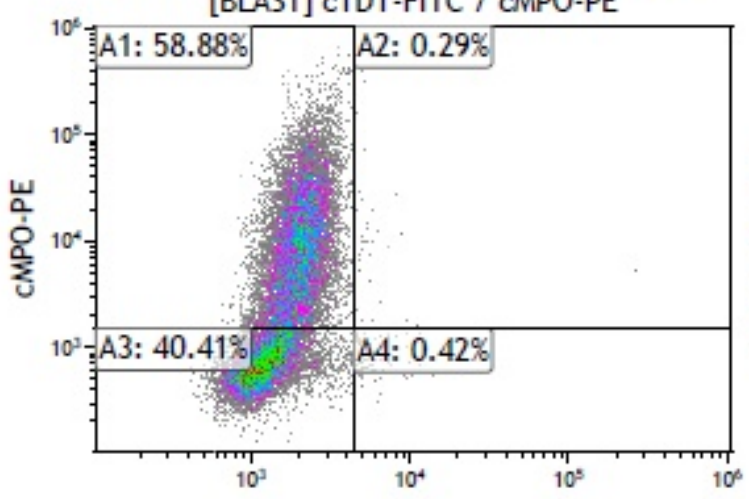

cTDT-FITC

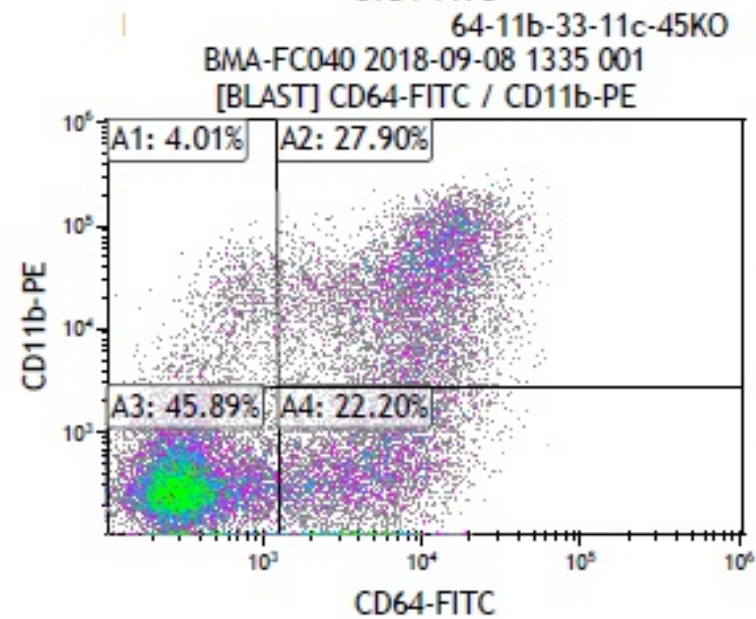

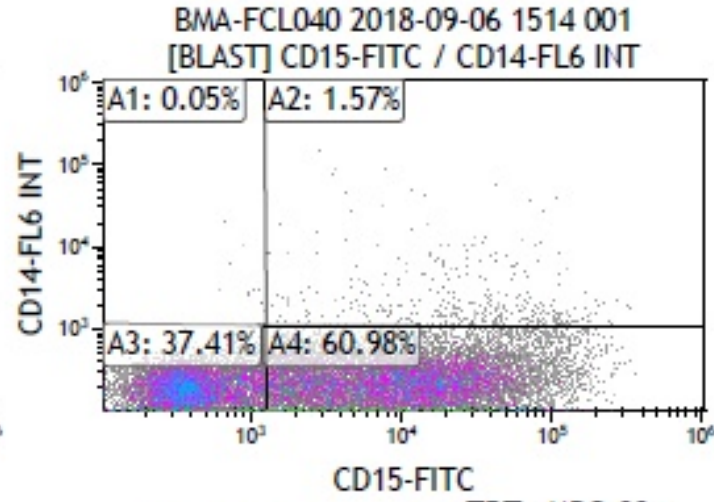

CTDT-cMPO-33-

c22-45KO BMA-FCL040 2018-09-06 1538

001

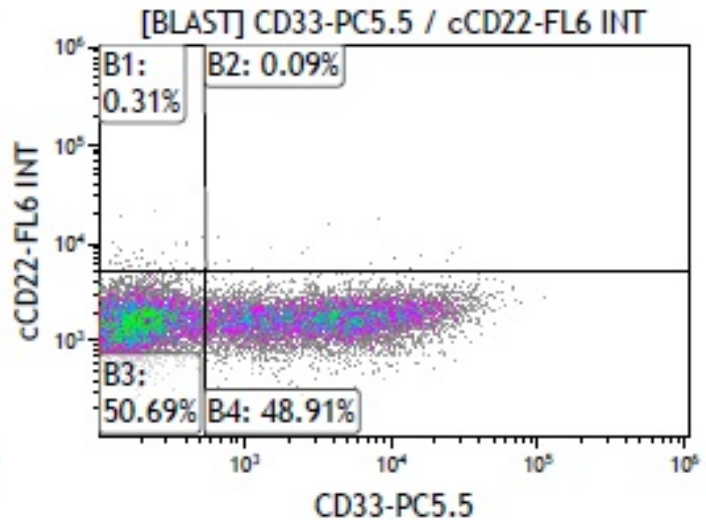

64-11b-33-11c-45KO BMA-FCO40 2018-09-08 1335001

[BLAST] CD33-PC5.5 / CD11C-FL6 INT

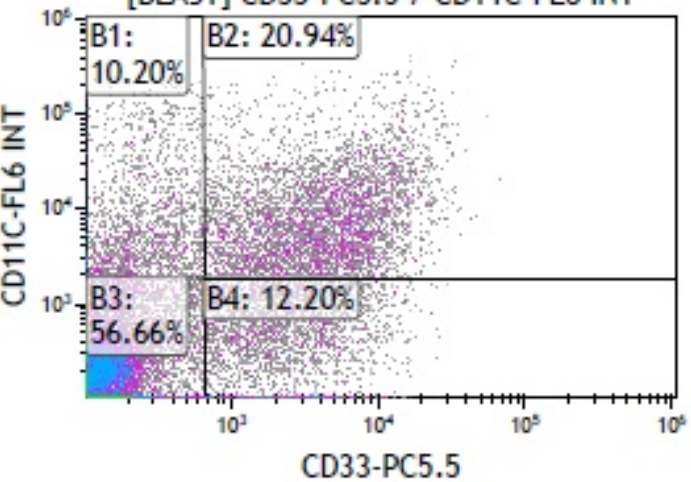

Figure 1. Flow cytometry analysis revealing mixed phenotype acute leukemia (MPAL) with 82\% blasts positive for CD19, CD33, CD15, CD11b,CD64, MPO, and CD79a.

the last antibiotic protocol. After a 6-month follow-up, the patient is still well and in CR ( $<2 \%$ blast cells).

\section{Discussion}

Herein we describe a case of MPAL showing specific and unusual biological and clinical features. MPAL is a rare high-risk disease, with an incidence among AL ranging from $1.5-2.4 \%[2,3]$. Thus, it is worth noting that the incidence of the MLL-AF4 rearrangement, evaluated in a very large series of AL cases [6] was strongly associated with adult ALL (274/333, 83\%), with very few cases $(<1 \%)$ among the 272 adult AML analyzed, making our case even more rare.
The lack of a specific prospective randomized trial likely due to the relative rarity of the disease and the consequent absence of evidencebased guidelines, make the therapeutic management of MPAL challenging [4,5]. However, it has become clear that ALL-directed induction chemotherapy provides a higher chance of achieving CR and a longer survival compared to an AML schedule [4,5]. Accordingly, a 7 -day pre-phase steroid treatment was administered. The low leukemic cell clearance achieved was considered an additional indicator of poor outcome and led us to switch towards AML-oriented treatment. The patient is currently in CR at six months follow-up. 


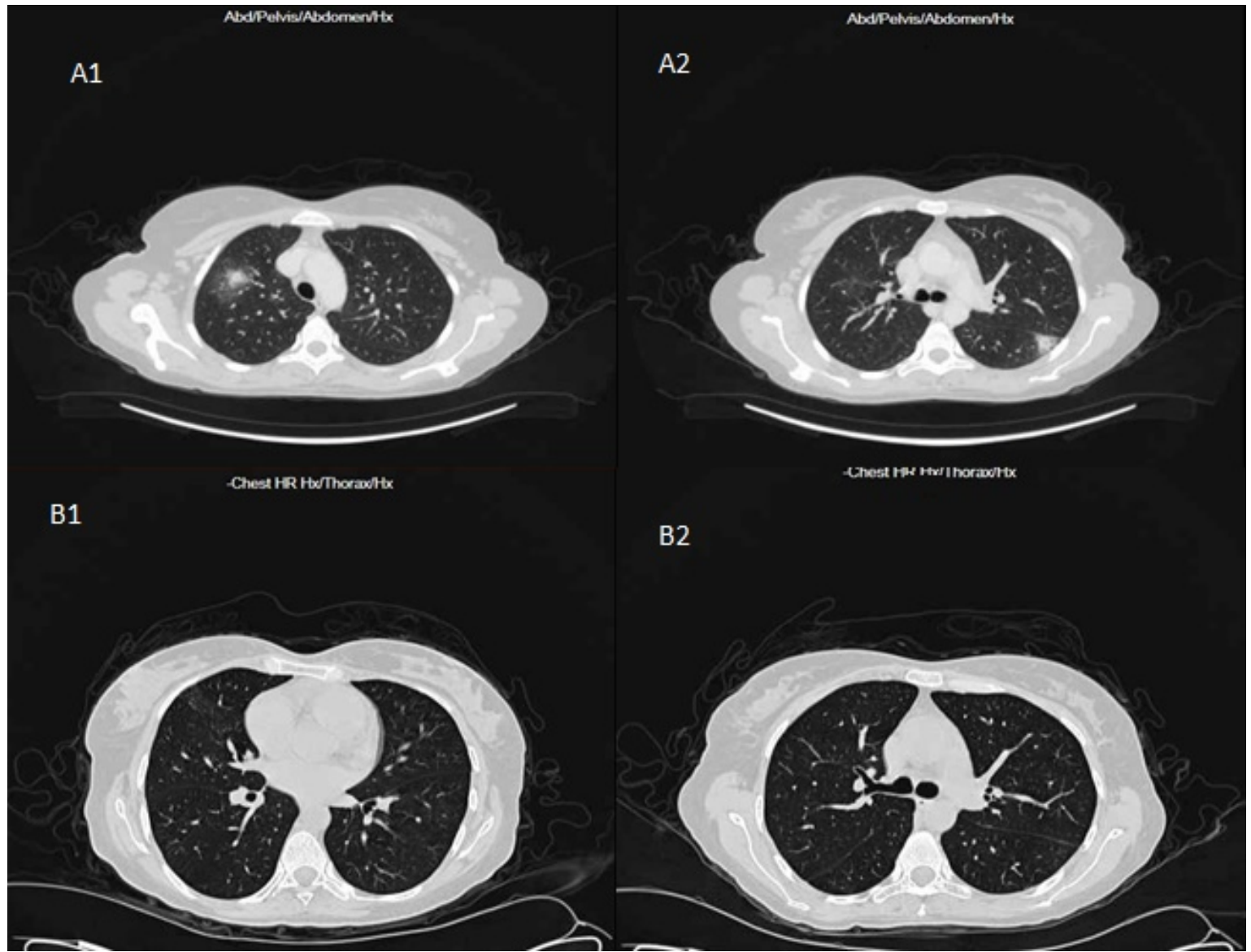

Figure 2. The high-resolution chest $\mathrm{CT}$ scan showed bilateral cavitary lesions (A1, A2), invasive fungal infection, and the radiological improvement (B1, B2) after treatment with voriconazole.

Notably, our patient had a high-risk infection profile, due to the pulmonary fungal infection, ESBL E.coli and CRE colonization, and ESBL E.coli and CRE induced post-consolidation febrile neutropenia episodes, which further complicated patient clinical outcome.

The patient was referred for allogeneic hematopoietic stem cell transplant (alloHSCT). In a large MPAL cohort, $6 \%$ and $11 \%$ of cases showed a trilineage phenotype and MLL-rearrangements, respectively [6]. Overall, 1-year transplant-related mortality was $14 \%$ with a 1 -year relapse rate of $31 \%$. However, no differences in overall survival were found among the diverse phenotype or among the MLLr and BCR-ABL high-risk cases.

\section{Conclusion}

In conclusion, we described a rare and difficult-to-treat MPAL case with additional molecular and infection-risk profiles. Although ALLoriented chemotherapy has been reported to be a superior treatment, steroid response could guide to switching to an AML-based protocol.

\section{References}

1. Arber DA, Orazi A, Hasserjian R, Thiele J, Borowitz MJ, et al. (2016) The 2016 revision to the World Health Organization classification of myeloid neoplasms and acute leukemia. Blood 127: 2391-2405. [Crossref]

2. Weinberg OK, Arber DA (2010) Mixed-phenotype acute leukemia: historical overview and a new definition. Leukemia 24: 1844-1851. [Crossref]

3. Khan M, Siddiqi R, Naqvi K (2018) An update on classification, genetics, and clinical approach to mixed phenotype acute leukemia (MPAL). Ann Hematol 97: 945-953. [Crossref]

4. Winters AC, Bernt KM (2017) MLL-Rearranged Leukemias-An Update on Science and Clinical Approaches. Front Pediatr 5: 4. [Crossref]

5. Matutes E, Pickl WF, Van't Veer M, Morilla R, Swansbury J, et al. (2011) Mixedphenotype acute leukemia: clinical and laboratory features and outcome in 100 patients defined according to the WHO 2008 classification. Blood 117: 3163-3171. [Crossref]

6. Wolach O, Stone RM (2017) Mixed-phenotype acute leukemia: Current challenges in diagnosis and therapy. Curr Opin Hematol 24: 139-145. [Crossref]

Copyright: (C2019 Qawasmeh N. This is an open-access article distributed under the terms of the Creative Commons Attribution License, which permits unrestricted use, distribution, and reproduction in any medium, provided the original author and source are credited. 